\title{
Anesthetic management during whole-lung lavage using lung ultrasound in a patient with pulmonary alveolar proteinosis: a case report
}

\author{
Jae Wan Jung ${ }^{1}$, Hyunho Lee ${ }^{2}$, Jimi Oh ${ }^{2}$ \\ ${ }^{1}$ Division of Pulmonary Medicine, Department of Internal Medicine, Wonkwang University Hospital, Iksan, Korea \\ ${ }^{2}$ Department of Anesthesiology and Pain Medicine, Ulsan University Hospital, University of Ulsan College of Medicine, Ulsan, Korea
}

Received: June 29, 2021

Revised: August 17, 2021

Accepted: August 21, 2021

Corresponding author:

Jimi Oh, MD

Department of Anesthesiology and

Pain Medicine, Ulsan University

Hospital, University of Ulsan College

of Medicine, 877

Bangeojinsunhwando-ro, Dong-gu,

Ulsan 44033, Korea

Tel: $+82-52-250-7248$

Fax: +82-52-250-7249

E-mail:0734952@uuh.ulsan.kr

\begin{abstract}
Pulmonary alveolar proteinosis (PAP) is an uncommon disease characterized by progressive accumulation of lipoprotein material in the lungs due to impaired surfactant clearance. Whole-lung lavage (WLL) is the current standard treatment and consists of sequential lavage of each lung to mechanically remove the residual material from the alveoli. Although WLL is considered safe, unexpected complications can occur. Moreover, due to the rarity of the disease itself, this procedure is unknown to many physicians, and management of intraoperative complications can be challenging for anesthesiologists. Lung ultrasound (LUS) provides reliable and valuable information for detecting perioperative pulmonary complications and, in particular, quantitation of lung water content. There have been reports on monitoring the different stages of controlled deaeration of the non-ventilated lung during WLL using LUS. However, it has been limited to non-ventilated lungs. Therefore, we report the use of LUS in WLL to proactively detect pulmonary edema in the ventilated lung and implement a safe and effective anesthesia strategy. Given the limited diagnostic tools available to anesthesiologists in the operating room, LUS is a reliable, fast, and noninvasive method for identifying perioperative pulmonary complications in patients with PAP undergoing WLL.
\end{abstract}

Keywords: Anesthesia; Extravascular lung water; One-lung ventilation; Pulmonary alveolar proteinosis; Ultrasonography

\section{Introduction}

Pulmonary alveolar proteinosis (PAP) is a rare disorder characterized by the accumulation of amorphous acellular lipoproteins in the alveoli, hindering gas exchange [1]. Whole-lung lavage (WLL) is the current standard treatment method consisting of sequential lavage of the affected lung through repeated filling and emptying cycles to remove excess alveolar phospholipids [2]. Although WLL is considered a safe and effective procedure for most patients with PAP, unexpected complications may arise. Knowledge of the physiological effects allows anesthesiologists to implement appropriate management [3].

Lung ultrasound (LUS) can help assess pulmonary and pleural pathologies in critically ill patients. It is now a standard technique for the early diagnosis of pulmonary edema in patients with heart failure, even in the subclinical stage [4]. There have been several reports on anesthesia management using LUS during WLL $[5,6]$. However, the use of LUS to monitor therapeutic procedures for both ventilated and non-ventilated lungs in the field of WLL is still in its infancy. 
We hypothesized that the previously validated LUS images could help identify aeration changes in ventilated and non-ventilated lungs during WLL. This modality may allow for early complication recognition, such as spillover into the ventilated lung, pleural effusion, and pulmonary edema, in addition to guiding anesthetic management and the troubleshooting of intraoperative complications in patients with PAP undergoing WLL. Herein, we report a case with the aim to reduce complications such as alveolar hyperinflation and systemic absorption of saline and overdistension of alveoli by early detection of pulmonary edema in the ventilated lung using these characteristics of LUS.

\section{Case}

A 54-year-old male patient with a height and weight of $180 \mathrm{~cm}$ and $78 \mathrm{~kg}$, respectively, presented to our hospital with a 2-month history of non-productive cough and progressive exertional dyspnea. The patient was a smoker (30 pack-years) without any other relevant medical history. Preoperative electrocardiography (ECG) and transthoracic echocardiography did not reveal any abnormalities. Chest radiography showed ill-defined bilateral opacities in the lower lung zones, and chest computed tomography showed extensive patchy ground-glass opacities in both lower lobes (Fig. 1). The left lung had more severe ill-defined opacities than the right lung. Preoperative pulmonary function testing results showed one-second forced expiratory volume $\left(\mathrm{FEV}_{1}\right)$ of $3.23 \mathrm{~L}$ (80\% of predicted), forced vital capacity (FVC) of $4.11 \mathrm{~L}$ (78\% of predicted), $\mathrm{FEV}_{1}$ / FVC ratio of $79 \%$, total lung capacity (TLC) of $5.40 \mathrm{~L}$ (75\% of predicted), and carbon monoxide diffusion capacity $\left(\mathrm{DL}_{\mathrm{CO}}\right)$ of $49 \%$. The patient exhibited a mild restrictive physiological pattern with decreased TLC and $\mathrm{DL}_{\mathrm{CO}}$. Cytological examination of the bronchoalveolar lavage fluid was suggestive of PAP, and pulmonologists planned for a WLL as first-line treatment.

Upon entering the operating room with a nasal cannula with 4 $\mathrm{L} / \mathrm{min} \mathrm{O}_{2}$, the patient was subjected to standard monitoring practices such as ECG, noninvasive blood pressure, bispectral index, and pulse oximetry. Before induction of anesthesia, all necessary equipment was checked and included $30 \mathrm{~L}$ of normal saline, a Y-piece connector, an irrigation set with clamps, a plastic container, and a rapid infusion system (RIS). Initial vital signs were stable at a blood pressure of 130/90 $\mathrm{mmHg}$, heart rate of 90 beats/min, respiratory rate of 16 breaths $/ \mathrm{min}$, and temperature of $36.2^{\circ} \mathrm{C}$. Initial arterial blood gas analysis (ABGA) showed a $\mathrm{pH}$ of 7.42, arterial partial pressure of carbon dioxide of $34 \mathrm{mmHg}$, arterial partial pressure of oxygen of $54 \mathrm{mmHg}$, bicarbonate concentration of $22.1 \mathrm{mmol} / \mathrm{L}$, and arterial oxygen saturation of $88 \%$ on room air. The alveolar-arterial oxygen difference was $53 \mathrm{mmHg}$, suggesting gas exchange issues. After preoxygenation, the anesthesiologist intravenously administered $1 \% \operatorname{propofol}(2 \mathrm{mg} / \mathrm{kg})$ and rocuronium $(0.6 \mathrm{mg} / \mathrm{kg})$. Total intravenous anesthesia with propofol and
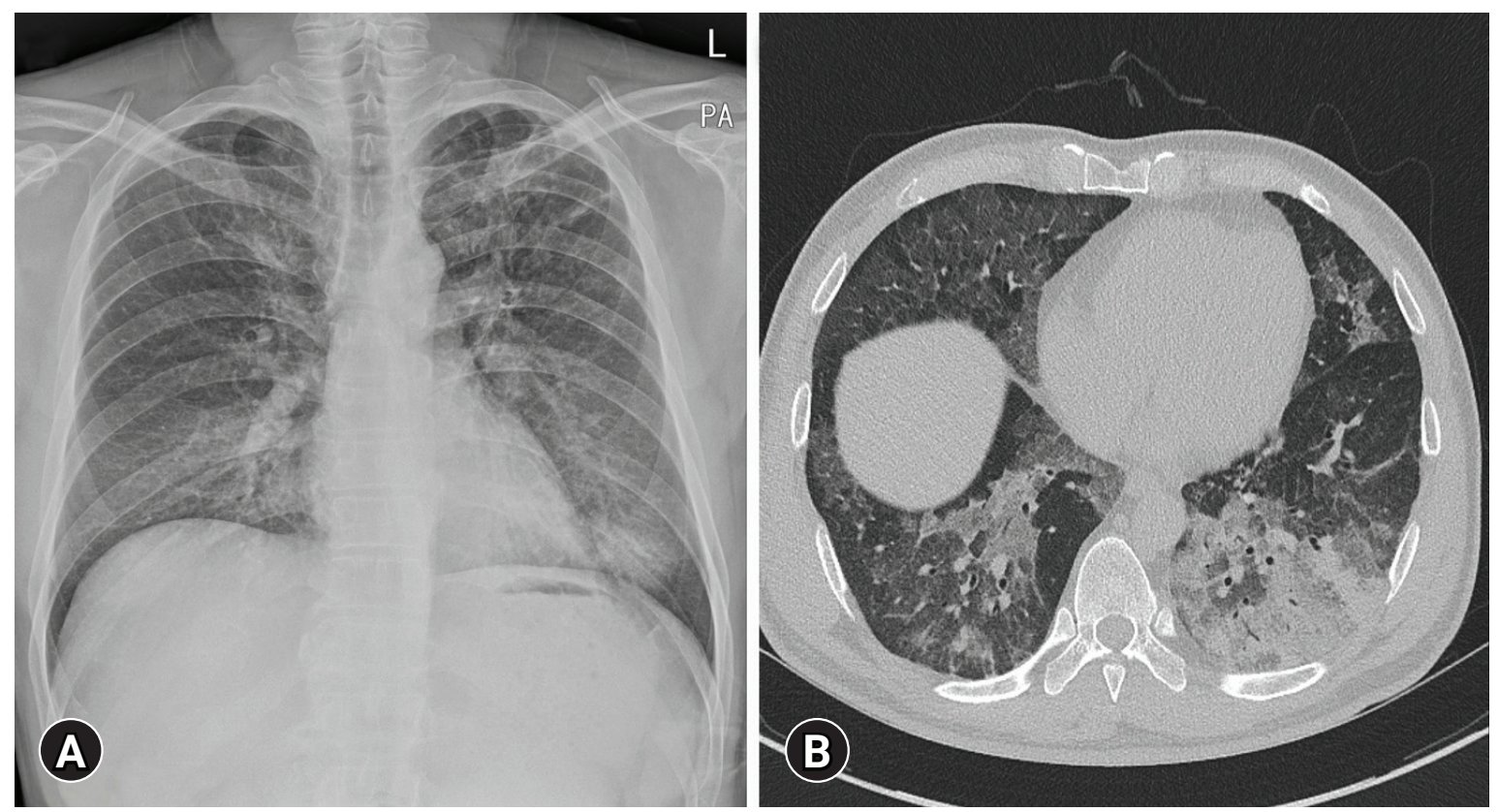

Fig. 1. (A) Chest radiograph shows ill-defined increased opacities in both lower lung zones. (B) High-resolution computed tomography scan shows ill-defined ground-glass opacities and consolidations with crazy-paving appearance in both lungs. 
remifentanil was maintained throughout the procedure. A 37-French (Fr) left-sided double-lumen endotracheal tube (DLT) was used for one-lung ventilation (OLV). We confirmed proper tube placement by auscultation of lung sounds and fiber-optic bronchoscopy. Because the left lung was more affected, a right-sided OLV was performed. A manual leak test was performed by immersing the non-ventilated left lung tube in saline while ventilating the right lung. Radial arterial and jugular venous catheters were used to continuously assess arterial blood gas and hemodynamic status during the perioperative period. The mechanical ventilation parameter setting was as follows: tidal volume was $6 \mathrm{~mL} \mathrm{~kg}^{-1}$ during two-lung ventilation (TLV), $4 \mathrm{~mL} / \mathrm{kg}^{-1}$ during $\mathrm{OLV}$, with 5 $\mathrm{cmH}_{2} \mathrm{O}$ positive end-expiratory pressure (PEEP) maintained throughout. Initially, WLL was started in the supine position. A Y-piece connected to the non-ventilated lung tube served as a link between the DLT and RIS. The warmed normal saline was then instilled into the left lung through the left tracheal lumen using the RIS at a rate of $<125 \mathrm{~mL} / \mathrm{min}$ with a $30^{\circ}$ head-up tilt (reverse Trendelenburg) position to facilitate the instillation (Fig. 2A). While draining the lavage fluid, the patient was placed in the headdown tilt (Trendelenburg) position (Fig. 2B), and the pulmonologist performed the process of chest percussion [1].

LUS was performed using a Versana Balance echograph and a 2-5 MHz convex array probe (GE Healthcare, Chicago, IL, USA).
The patient was scanned in the supine position by a single anesthesiologist (JO). Three regions (phrenic point, upper and lower BLUE points) were identified bilaterally in each hemithorax according to the bedside LUS in emergency (BLUE) protocol (Fig. 3) [7]. Images were obtained at four intervals: (1) baseline (before anesthetic induction), (2) end lavage (after the introduction of the last bolus of normal saline into the non-ventilated lung), (3) reven-

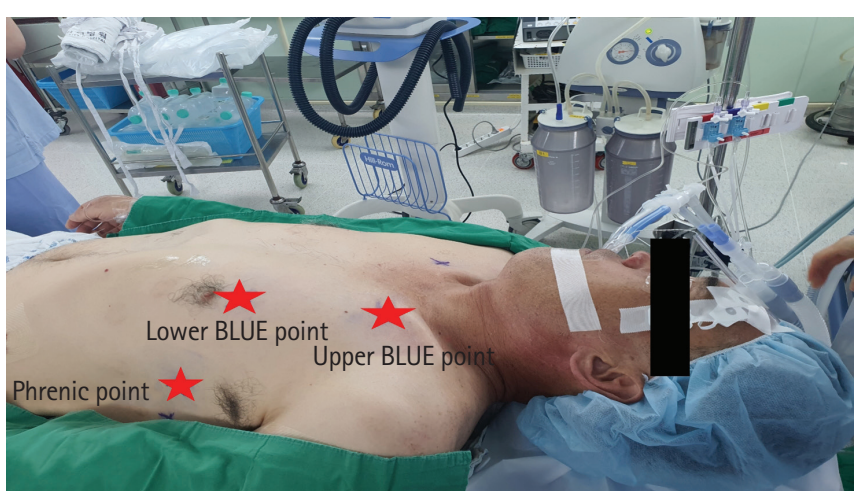

Fig. 3. To ensure the reproducibility of ultrasound acquisition during the entire procedure, the exact point of the lung ultrasound examination was marked on the skin. Three regions (phrenic point, upper and lower BLUE points) were identified bilaterally in each hemithorax according to the bedside lung ultrasound in emergency (BLUE) protocol.
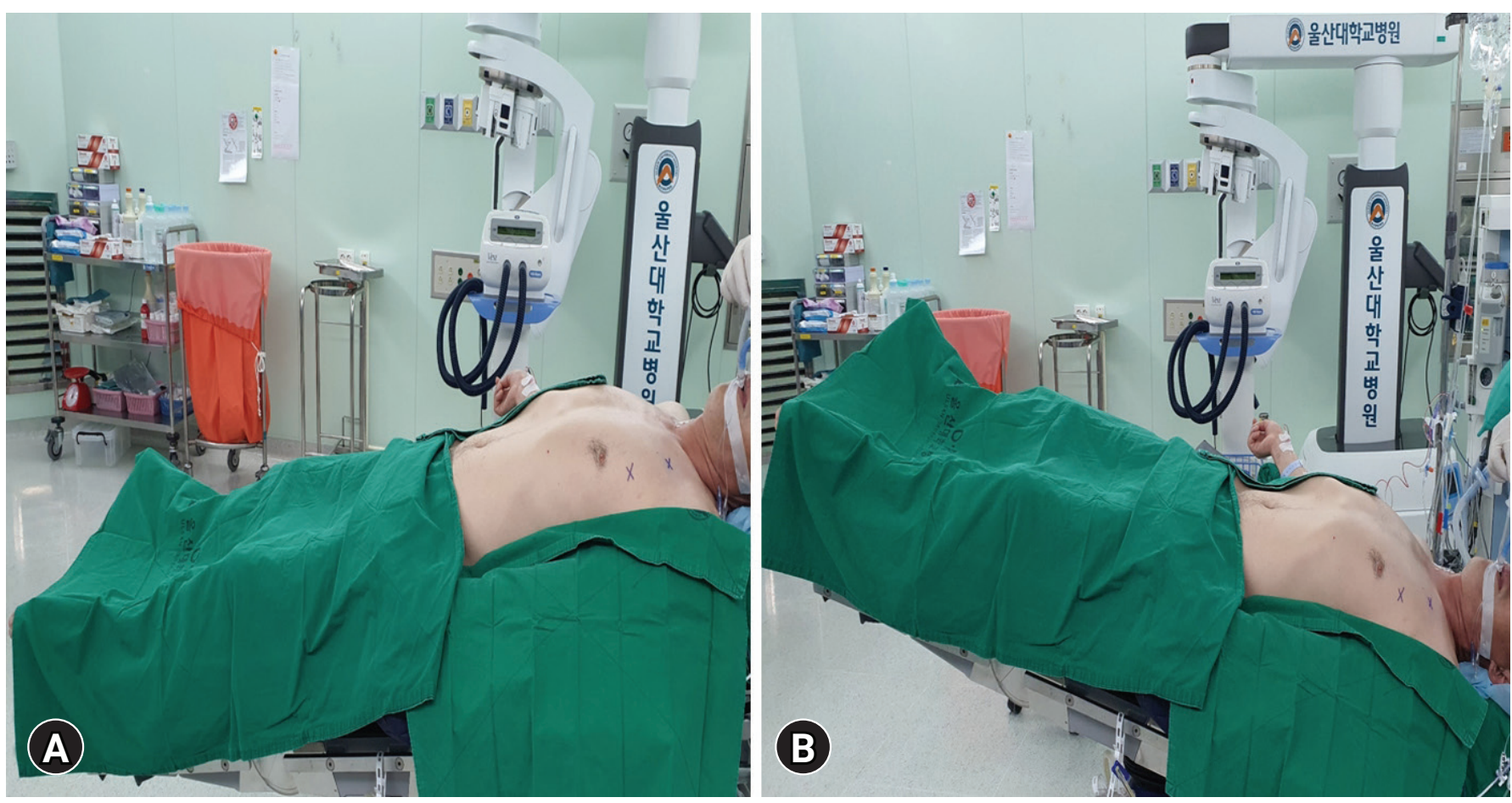

Fig. 2. Picture of patient's position during whole-lung lavage. (A) During inflow of lavage fluid, the patient was positioned in the reverse Trendelenburg to facilitate the instillation. (B) While draining the lavage fluid, the patient was placed in the Trendelenburg position. 
tilation (after reinflation of the non-ventilated lung), and (4) pre-extubation (immediately before extubation). Six points were explored, and ABGA was performed in each of the four steps. Each finding was classified into three patterns according to previously reported LUS semiotics (Table 1) [2]. The lavage procedure was terminated when a tissue pattern (No. 3 in Table 1) appeared in all areas of the non-ventilated lung or three or more B-lines (No. 2 in Table 1) appeared in the ventilated lung without waiting for the fluid to overflow into the endotracheal tube. At the same time, serial ABGAs were performed to monitor hypoxemia, hypercapnia, acidosis, and electrolyte imbalance (Table 2). At the 9th cycle, an imbalance of more than $100 \mathrm{~mL}$ between infusion and drained volumes, as well as an increased number of B-lines of the ventilated lung, was observed compared to the previous cycle (II in Fig. 4). We managed with an adequate PEEP of $7 \mathrm{cmH}_{2} \mathrm{O}$ and negative water balance using intravenous diuretics $(0.5 \mathrm{mg} / \mathrm{kg}$ intravenous

Table 1. Patterns describing lung ultrasound findings during whole-lung lavage

\begin{tabular}{|c|c|c|}
\hline No. & Pattern & Description \\
\hline 1 & Normal/nearly normal pattern & $\begin{array}{l}\text { Pleural line, pleural sliding, A-lines (normal pattern), or B-lines in number }<3 \text { per lung ultrasound scan } \\
\text { (nearly normal pattern) }\end{array}$ \\
\hline 2 & Alveolar-interstitial syndrome & $\begin{array}{l}\text { B-lines in number } \geq 3 \text { per lung ultrasound scan, more or less crowded, up to complete coalescence of } \\
\text { these artifacts (the so-called "white lung") }\end{array}$ \\
\hline 3 & Alveolar consolidation & $\begin{array}{l}\text { Tissue-like pattern, with precise anatomical boundaries, no dimensional change throughout the respiratory } \\
\text { cycle and variable depth extension }\end{array}$ \\
\hline
\end{tabular}

Table 2. Results of arterial blood gas analysis during left lung lavage

\begin{tabular}{|c|c|c|c|c|c|c|c|}
\hline Variable & Basal & OLV 5 & OLV 30 & End lavage & Reventilation & Pre-extubation & Post-extubation \\
\hline $\mathrm{pH}$ & 7.42 & 7.3 & 7.27 & 7.33 & 7.31 & 7.43 & 7.42 \\
\hline $\mathrm{PaO}_{2}(\mathrm{mmHg})$ & 54 & 72 & 76 & 59 & 64 & 107 & 81 \\
\hline $\mathrm{PaCO}_{2}(\mathrm{mmHg})$ & 34 & 52 & 49 & 44 & 44 & 34 & 35 \\
\hline $\mathrm{HCO}_{3}^{-}(\mathrm{mEq} / \mathrm{L})$ & 22.1 & 25.6 & 22.5 & 23.2 & 22.2 & 22.6 & 22.7 \\
\hline $\mathrm{SaO}_{2}(\%)$ & 88 & 92 & 93 & 88 & 90 & 98 & 96 \\
\hline $\mathrm{FiO}_{2}$ & 0.2 & 1.0 & 1.0 & 1.0 & 0.6 & 0.3 & 0.2 \\
\hline $\mathrm{P} / \mathrm{F}$ ratio & 257 & 72 & 76 & 59 & 107 & 357 & 386 \\
\hline
\end{tabular}

$\mathrm{OLV}$, one-lung ventilation; OLV 5 , within 5 minutes of $\mathrm{OLV} ; \mathrm{OLV} 30$, within 30 minutes of $\mathrm{OLV} ; \mathrm{PaO}_{2}$, arterial partial pressure of oxygen; $\mathrm{PaCO}$, arterial partial pressure of carbon dioxide; $\mathrm{HCO}_{3}{ }^{-}$, bicarbonate; $\mathrm{SaO}_{2}$, arterial oxygen saturation; $\mathrm{FiO}_{2}$, fraction of inspired oxygen; $\mathrm{P} / \mathrm{F}$ ratio, $\mathrm{PaO}_{2} / \mathrm{FiO}_{2}$ ratio.

Ventilated lung

Non-ventilated lung (lavaged lung)
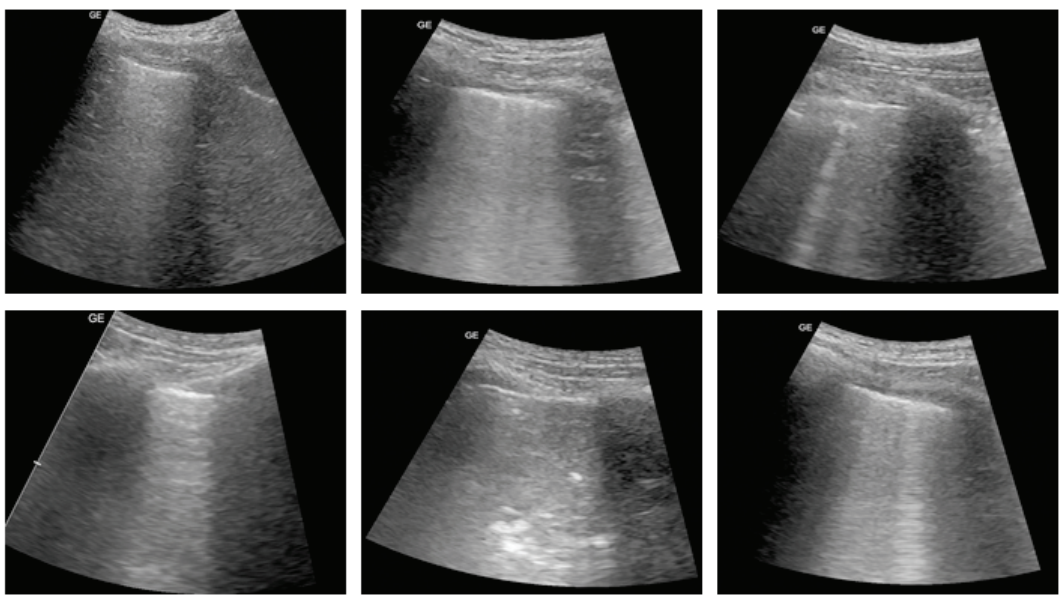

II

End lavage

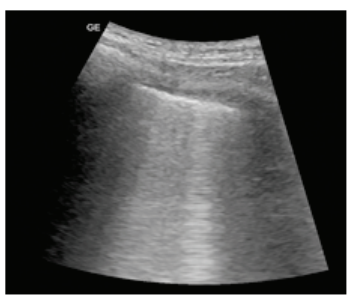

III

Reventilation
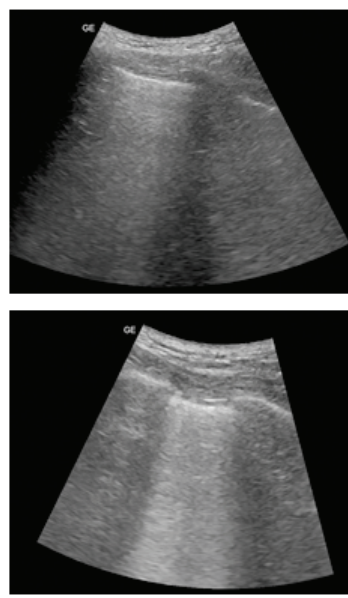

IV

Pre-extubation

Fig. 4. The typical sequence of ultrasound findings of the lung. Our lavage procedure was terminated when a tissue pattern (pattern 3, II) appeared in all areas of the non-ventilated lung and/or three or more B-lines (pattern 2, II) appeared in the ventilated lung. Reventilation was associated with reduced B-lines in the ventilated lung and a reappearance of pattern 2 (alveolar-interstitial syndrome) in the lavaged lung. Prolonged ventilation with PEEP (18 hours later, ventilation with $10 \mathrm{cmH}_{2} \mathrm{O}$ PEEP) resulted in the return of pattern 1 (normal pattern) in both lungs. PEEP, positive end-expiratory pressure. 
furosemide). The procedure was then terminated. Subsequently, TLV was initiated. Several B-lines were observed in the lavaged lung, whereas the ventilated lung showed a regular pattern with a marked decrease (III in Fig. 4). The total normal saline administered was $9.8 \mathrm{~L}$, and $8.9 \mathrm{~L}$ saline was retrieved from the left lung. Intraoperative fluid management was performed with intermittent confirmation of the volume status by qualitative methods such as visual estimation using transthoracic echocardiography because of its technical complexity and time-consuming nature. The total volume of intravenously infused crystalloid solution administered to the patient during the WLL procedure was $1,850 \mathrm{~mL}$, and the total urine output during the procedure was $450 \mathrm{~mL}$. The DLT was exchanged for a $7.0 \mathrm{~mm}$ single lumen, and the patient was transferred to the intensive care unit. The next day, the patient was extubated.

Manual chest physiotherapy was continued postoperatively. The symptoms improved clinically with a saturation of $\geq 94 \%$ on room air (Table 2), and he was discharged 1 week after the procedure.

\section{Discussion}

Our case report demonstrates the feasibility of using LUS during all phases of WLL. Although there are several reports of using LUS for monitoring procedures during WLL, little is known about the use of LUS in terminating the WLL process to prevent flooding of the ventilated lung. We identified an increase in the number of B-lines through LUS, suggesting pulmonary edema in the ventilated lung and efficiently determining the termination of WLL.

PAP is a diffuse lung disease characterized by the accumulation of amorphous, periodic acid-Schiff-positive lipoprotein material in the distal air spaces [8]. Treatment depends on the severity of the disease. The indications for WLL are decreased lung function, radiographic signs of disease progression, poor alveolar gas exchange, and worsening of respiratory symptoms. The contraindications are uncorrectable clotting disorders, high anesthetic risk, and cardiopulmonary instability [3]. In our case, the patient underwent WLL because of the progressive worsening of respiratory symptoms. The left lung with a more severe disease was lavaged first in our patient, whereas the right lung was lavaged under general anesthesia after 1 month.

Although WLL is a widely used therapeutic procedure for patients with PAP, the short-term or long-term outcomes of the procedure are not well described. In general, approximately 10 to 15 lavages are performed to remove lavage effluent from each lung, each requiring 1 to $1.5 \mathrm{~L}$ of warm saline [9]. WLL is terminated when the lavage effluent appears clear on visual inspection of the protein content of the sample [3]. Specifically, if the patient's hemodynamic status and oxygenation are tolerable, this process is re- peated until the lavage effluent is washed out satisfactorily. Although the lavage method seems simple, the clinical results reported so far are diverse, ranging from complete resolution to death due to respiratory failure or pneumonia [10]. Some authors have mentioned delayed improvement or temporary worsening of lung function after WLL [11]. In addition, Huber et al. [12] described changes in respiratory mechanics and alveolar morphology after WLL in dogs. This procedure has also been shown to remove large amounts of surface-active substances [13] and other proteins [14]. In other words, saline lavage of the lungs until the lavage effluent is clear may not necessarily be a good thing. Moreover, the procedure of WLL is associated with complications such as pneumothorax, pleural effusion, hydropneumothorax, mediastinal shift, and increased intrathoracic and central venous pressures due to excessive lavage fluid, leading to hypotension. In particular, the risk of pulmonary edema in the ventilated lung is significantly higher in prolonged procedures such as WLL. Several mechanisms can cause pulmonary edema in the ventilated lung during the WLL procedure. Intraoperative fluid overload or lavage fluid leakage into the ventilated lung is a common cause. In addition, it is believed that changes in hydrostatic forces in the pulmonary microcirculation or activation of proinflammatory cytokines from the collapsed lung may cause pulmonary edema in the ventilated lung [15]. Therefore, since the non-ventilated lung is fully consolidated during WLL, mild pulmonary edema of the ventilated lung can significantly impact the prognosis of patients with preexisting PAP. This is an important reason for the early detection of pulmonary edema that occurs in ventilated lungs during the WLL procedure using LUS. Indeed, pulmonary edema by LUS has been evaluated in traumatic brain injury, acute respiratory distress syndrome, chronic renal failure, and cardiac surgery, with good sensitivity (97\%) and specificity (98\%) [4,16-18]. However, it has rarely been evaluated in the WLL procedure, especially in ventilated lungs, which may represent a potentially unique problem due to rapidly changing fluid dynamics. In our case, we confirmed an increase in the number of B-lines compared to baseline in ventilated lungs via LUS, which enabled early detection of pulmonary edema in ventilated lungs.

LUS has developed dramatically in the past decade, and its advantages include a lack of radiation and bedside accessibility. It has become a powerful tool for managing critically ill patients with acute respiratory failure, including diagnostic assessment, identifying the cause of acute gas exchange deterioration during ventilation support, and investigating weaning failure [19]. WLL represents a typical human model of lung aeration changes, similar to various pathological conditions in critically ill patients. The overall course of WLL, which includes OLV and progressive alveolar overflow, is quite similar to resorption atelectasis and lung consolidation. In 
this case report, LUS reliably monitored and recorded changes in lung aeration. Starting from a nearly regular pattern to apparent changes in lung deaeration throughout WLL were associated with significant changes in LUS findings in both ventilated and non-ventilated lungs. In the non-ventilated lung, the transition from normal to the alveolar-interstitial pattern was achieved during OLV, and continuous alveolar flooding promoted the transition to a consolidation pattern. Reventilation was more likely to result in a pattern of alveolar-interstitial syndrome due to residual water in the lavaged lung. In the ventilated lung, the number of B-lines progressively increased regularly as the unventilated one overflowed with saline. We considered this to be the endpoint of WLL. After adequate PEEP and negative water balance, the number of B-lines in the ventilated lung was significantly reduced, and it reappeared in a normal pattern. As in previous studies, terminating WLL when the lavage effluent is clear can be very subjective. In addition, because there are no randomized trials or formal prospective studies on the WLL procedure, it is difficult to determine the impact of current lavage methods on patient prognosis. In our case, as mentioned above, the endpoint of WLL was defined as the appearance of pulmonary edema in the ventilated lung. Our patient underwent similar anesthetic management using LUS for WLL in the contralateral lung after 1 month. Serial WLL resulted in clinical and physiological improvement in the patient. According to previous studies, and in 55\% of patients with PAP, repeated lavage every 6 to 12 months may be required [20]. Moreover, in a significant number of patients, it is often impossible to implement continuous WLL due to hypoxemia or poor clinical conditions. Therefore, given the high burden of complications in patients undergoing WLL, early identification and management of complications using simple and convenient tools such as LUS would be valuable. In addition, keeping the ventilated lung stable can be just as important as thoroughly cleaning the non-ventilated lung. Clarifying the relationship between the WLL procedure and worse outcomes may help to identify therapeutic strategies to avoid complications after WLL. Further studies are required to examine this mechanism.

Our case report focused on anesthetic management using LUS to identify intraoperative complications often encountered during WLL. In particular, it is essential to monitor non-ventilated and ventilated lungs using LUS to prevent complications during the procedure. This case provides insight into the procedure and usefulness of LUS from an anesthesiologist's perspective and potential intraoperative complications. The availability of LUS during the WLL procedure may enable early diagnosis of complications and improve patient prognosis.

\section{Notes}

\section{Ethical statements}

This study was approved by the Institutional Review Board (IRB) of Ulsan University Hospital (IRB No: 2021-06-020). Written informed consent was obtained for the publication of this case report and accompanying images.

\section{Conflicts of interest}

No potential conflict of interest relevant to this article was reported.

\section{Author contributions}

Conceptualization: JWJ, JO; Formal analysis, Funding acquisition, Supervision, Validation: JO; Methodology: HL, JO; Project administration: HL; Writing-original draft: JWJ, HL, JO; Writing-review \& editing: JWJ, JO.

\section{ORCID}

Jae Wan Jung, https://orcid.org/0000-0003-3816-9867

Hyunho Lee, https://orcid.org/0000-0001-7963-5006

Jimi Oh, https://orcid.org/0000-0002-2365-0642

\section{References}

1. Ra SW, Park SE, Lee HK, Han IS, Park SH. Whole lung lavage using a rapid infusion system to treat a patient with pulmonary alveolar proteinosis. Yeungnam Univ J Med 2020;37:67-72.

2. Via G, Lichtenstein D, Mojoli F, Rodi G, Neri L, Storti E, et al. Whole lung lavage: a unique model for ultrasound assessment of lung aeration changes. Intensive Care Med 2010;36:999_ 1007.

3. Mata-Suarez SM, Castro-Lalín A, Mc Loughlin S, De Domini J, Bianco JC. Whole-lung lavage-a narrative review of anesthetic management.J Cardiothorac Vasc Anesth 2020 Dec 5 [Epub]. https://doi.org/10.1053/j.jvca.2020.12.002.

4. Picano E, Pellikka PA. Ultrasound of extravascular lung water: a new standard for pulmonary congestion. Eur Heart J 2016; 37:2097-104.

5. Misra S, Das PK, Bal SK, Elayat A, Sahoo S, Dahl AB, et al. Therapeutic whole lung lavage for alveolar proteinosis. J Cardiothorac Vasc Anesth 2020;34:250-7.

6. Ramachandran P, Chaudhury A, Devaraj U, Maheshwari KU, D'Souza G. Monitoring whole-lung lavage using lung ultrasound: the changing phases of the lung. Lung India 2018;35: 350-3.

7. Lichtenstein DA. BLUE-protocol and FALLS-protocol: two 
applications of lung ultrasound in the critically ill. Chest 2015; 147:1659-70.

8. Kumar A, Abdelmalak B, Inoue Y, Culver DA. Pulmonary alveolar proteinosis in adults: pathophysiology and clinical approach. Lancet Respir Med 2018;6:554-65.

9. Campo I, Luisetti M, Griese M, Trapnell BC, Bonella F, Grutters J, et al. Whole lung lavage therapy for pulmonary alveolar proteinosis: a global survey of current practices and procedures. Orphanet J Rare Dis 2016;11:115.

10. Seymour JF, Presneill JJ. Pulmonary alveolar proteinosis: progress in the first 44 years. Am J Respir Crit Care Med 2002;166: 215-35.

11. Case records of the Massachusetts General Hospital. Weekly clinicopathological exercises. Case 34-1974. N Engl J Med 1974;291:464-9.

12. Huber GL, Edmunds LH Jr, Finley TN. Effect of experimental saline lavage on pulmonary mechanics and morphology. Am Rev Respir Dis 1971;104:337-47.

13. McClenahan JB. Pulmonary alveolar proteinosis. Arch Intern Med 1974;133:284-7.

14. Rupp GH, Wasserman K, Ogawa M, Heiner DC. Bronchopulmonary fluids in pulmonary alveolar proteinosis. J Allergy Clin Immunol 1973;51:227-37.
15. Rogers RM, Szidon JP, Shelburne J, Neigh JL, Shuman JF, Tantum KR. Hemodynamic response of the pulmonary circulation to bronchopulmonary lavage in man. N Engl J Med 1972;286: 1230-3.

16. Anile A, Russo J, Castiglione G, Volpicelli G. A simplified lung ultrasound approach to detect increased extravascular lung water in critically ill patients. Crit Ultrasound J 2017;9:13.

17. Agricola E, Bove T, Oppizzi M, Marino G, Zangrillo A, Margonato A, et al. "Ultrasound comet-tail images": a marker of pulmonary edema: a comparative study with wedge pressure and extravascular lung water. Chest 2005;127:1690-5.

18. Enghard P, Rademacher S, Nee J, Hasper D, Engert U, Jörres A, et al. Simplified lung ultrasound protocol shows excellent prediction of extravascular lung water in ventilated intensive care patients. Crit Care 2015;19:36.

19. Leblanc D, Bouvet C, Degiovanni F, Nedelcu C, Bouhours G, Rineau E, et al. Early lung ultrasonography predicts the occurrence of acute respiratory distress syndrome in blunt trauma patients. Intensive Care Med 2014;40:1468-74.

20. Goldstein LS, Kavuru MS, Curtis-McCarthy P, Christie HA, Farver C, Stoller JK. Pulmonary alveolar proteinosis: clinical features and outcomes. Chest 1998;114:1357-62. 